\title{
The new affordances in the home environment for motor development - infant scale (AHEMD-IS): Versions in English and Portuguese languages
}

\author{
Priscila M. Caçola ${ }^{1}$, Carl Gabbard ${ }^{2}$, Maria I. L. Montebelo ${ }^{3}$, \\ Denise C. C. Santos ${ }^{4}$
}

\begin{abstract}
The home environment has been established as a crucial factor for motor development, especially in infants. Exploring the home environment can have significant implications for intervention, as it is common practice in physical therapy to have professionals advise patients on home activities. Since 2010, our group has been working on the development of the Affordances in the Home Environment for Motor Development - Infant Scale (AHEMD-IS), a parental self-reporting instrument designed to assess the quality and quantity of factors (affordances) in the home environment. In Brazil, the instrument has been translated as "Affordances no Ambiente Domiciliar para o Desenvolvimento Motor - Escala Bebê”, and it has been extensively used in several studies that address infant development. These studies in Brazil and other parts of the world highly recommended the need for a normative sample and standardized scoring system. A description of the study that addressed that need, along with the English version of the questionnaire and score sheets, was recently published in the well-known and respected journal Physical Therapy. Our intent with the present short communication is to notify Brazilian investigators and clinicians of this latest update so they can download the new instrument, as well as present the Brazilian (Portuguese) version of the AHEMD-IS along with its scoring system.
\end{abstract}

Keywords: affordances; motor development; infants; home.

\section{BULLET POINTS}

- The home environment is crucial for infant motor development.

- We validated the Affordances in the Home Environment for Motor Development - Infant Scale (AHEMD-IS).

- We present the standardized version and scoring system of the instrument.

\section{HOW TO CITE THIS ARTICLE}

Caçola PM, Gabbard C, Montebelo MIL, Santos DCC. The new affordances in the home environment for motor development - infant scale (AHEMD-IS): Versions in English and Portuguese languages. Braz J Phys Ther. 2015 Nov-Dec; 19(6):507-525. http://dx.doi.org/10.1590/bjpt-rbf.2014.0112

The home environment has been established as a crucial factor for motor development, especially in infants ${ }^{1}$. Exploring the home environment can have significant implications for intervention, as it is common practice in physical therapy to have professionals advise patients on home activities. In general, such recommendations involve how to utilize different aspects of home space, toys, stimulation, and activities that are part of an infant's everyday life ${ }^{2}$. Since 2010, our group has been working on the development of the Affordances in the Home Environment for Motor Development - Infant Scale (AHEMD-IS), a parental self-reporting instrument designed to assess the quality and quantity of factors (affordances) in the home environment. More specifically, the instrument addresses the dimensions of Physical Space, Variety of Stimulation, and Play Materials in the home that are conducive to enhancing motor development of infants aged 3 to 18 months $^{1}$. Since its first publication in 2011 (as a descriptive tool with preliminary validity guidelines), the AHEMD-IS has gained in popularity as a clinical tool and as a research outcome measure ${ }^{3-8}$.

In Brazil, the instrument has been translated as "Affordances no Ambiente Domiciliar para o Desenvolvimento Motor - Escala Bebê", and it has been extensively used in several studies that address

\footnotetext{
${ }^{1}$ Developmental Motor Cognition Lab, Department of Kinesiology, The University of Texas at Arlington, Arlington, TX, USA

${ }^{2}$ Child Motor Development Lab, Department of Health and Kinesiology, Texas A\&M University, College Station, TX, USA

${ }^{3}$ Faculdade de Ciências Exatas e da Natureza, Universidade Metodista de Piracicaba (UNIMEP), Piracicaba, SP, Brazil

${ }^{4}$ Programa de Pós-Graduação em Fisioterapia e em Ciências do Movimento Humano, Faculdade de Ciências da Saúde, Universidade Metodista de Piracicaba (UNIMEP), Piracicaba, SP, Brazil

Received: Apr. 29, 2015 Revised: June 03, 2015 Accepted: June 15, 2015
} 
infant development. For example, it was an important tool in a study exploring the role of biological and environmental factors in infant development, supporting the argument that environmental factors are associated with infant motor development ${ }^{3}$. Similarly, the AHEMD-IS has been recently added as a supporting tool in a major study designed to measure the effects of intrauterine growth restriction ${ }^{4}$. From other perspectives of infant development, a study has established that the availability of fine- and gross-motor toys in the home environment represents the real use of these opportunities (affordances) by infants 5 . Furthermore, it appears that all dimensions of the AHEMD-IS are highly influenced by socioeconomic status (SES) ${ }^{6}$ with the exception of Variety of Stimulation ${ }^{7}$, and there are differences in home affordances when exploring two diverse areas of Brazil ${ }^{8}$.

With that said, studies in Brazil and other parts of the world that utilized the AHEMD-IS highly recommended the need for a normative sample and standardized scoring system. A description of the study that addressed that need, along with the English version of the questionnaire and score sheets, was recently published in the well-known and respected journal Physical Therapy ${ }^{1}$. Our intent with the present short communication is to notify Brazilian investigators and clinicians of this latest update so they can download the new instrument, as well as present the Brazilian (Portuguese) version of the AHEMD-IS along with its scoring system. The whole process of further development and validation of the instrument included three phases: (1) use of expert opinion for content validity; (2) administration to a large Brazilian sample and testing of reliability, consistency, and floor and ceiling effects; and (3) re-testing of internal consistency and determination of interpretability for the scoring system.

As described in the Physical Therapy article ${ }^{1}$, Phase 1 (expert opinion of content) resulted in an average agreement of $95 \%$ across all criteria and a reduction of items (46-41). Phase 2 results showed ICC values of .99 for interrater and .95 for intrarater reliability, with Cronbach's alpha values ranging between .64 and .82 for the dimensions and a Total value of .82 (.78-.86) for internal consistency. Ceiling effects were found on three questions of the Inside Space dimension and three in Variety of Stimulation. These results demonstrated the need for reduction in total items (41 to 35) and combination of the Space sections. Results in Phase 3 revealed an internal consistency of .77 (.73-.80) for Total Affordances in the home. Considering that the quoted alphas for each dimension ranged from 0.66 to 0.76 , with 3 out of the 4 dimensions with an alpha $>0.7$, the questionnaire can be considered as having satisfactory internal validity. Interpretability of the scores was based on quartile analysis and creation of four descriptive categories (Less than Adequate, Moderately Adequate, Adequate, and Excellent) that represent the quality and quantity of home affordances for motor development and its respective dimensions.

Steps in the revision confirmed that the AHEMD-IS is a valid and reliable instrument for the assessment of infants 3 to 18 months of age. The instrument now consists of 35 items divided into 4 dimensions (Physical Space, Variety of Stimulation, Fine-Motor Toys, and Gross-Motor Toys) and a Total score that can be categorized into 4 descriptions of home motor affordances (scoring system): Less than adequate, Moderately Adequate, Adequate, and Excellent. The results suggest that this tool can be a useful instrument for measuring the quantity and quality of affordances in the home environment that are conducive to infant motor development. Researchers can utilize this instrument to account for this very important contributor to infant motor development, while physical therapists can use it for assessment and recommendations for intervention, along with advising parents on home activities.

For a detailed description of the latest development and validation of the AHEMD-IS, readers can visit the journal website ${ }^{1}$. The English version of the questionnaire and score sheet is also published in the article in the appendix. The questionnaire and score sheet versions in Brazilian Portuguese are included as an appendix of this communication and are available for download ${ }^{9}$. There were few modifications in the Brazilian version, e.g., there are no longer references to ethnic background, the educational system has been adjusted to Brazilian parameters, and further observations regarding the risks of using baby walkers and the safety of stairs were added. The final instrument is provided in Appendix 1. Appendix 2 provides the separate score sheets for infants aged 3 to 11 months and infants aged 12 to 18 months, with examples of how to use the AHEMD-IS to improve the home environment. More information about the instrument can also be found on the same website ${ }^{9}$, in both English and Portuguese. We believe that the AHEMD-IS is an important instrument to account for the home as one of the environmental factors influencing infant motor development, and it has noteworthy research ${ }^{10}$ 
and clinical promise. That promise includes insight to the influence of home motor affordances not only on motor development, but also on future cognitive and social behavior. The Portuguese version of the instrument presented here is the official translation of the latest version of the AHEMD-IS. Dr. Caçola and Dr. Santos would appreciate hearing about data collected using the AHEMD-IS.

\section{- Acknowledgements}

This work was partially supported by CNPq-Brazilian Council of Technological and Scientific Development (Process 486077/2011-0 and 308903/2012-9).

\section{References}

1. Caçola PM, Gabbard C, Montebelo MIL, Santos DCC. Further Development and Validation of the Affordances in the Home Environment for Motor Development-Infant Scale (AHEMD-IS). Phys Ther. 2015;95(6):901-23. http:// dx.doi.org/10.2522/ptj.20140011. PMid:25524875.

2. Caçola P, Gabbard C, Santos DCC, Batistela AC. Development of the affordances in the home environment for motor development - infant scale. Pediatr Int. 2011;53(6):8205. http://dx.doi.org/10.1111/j.1442-200X.2011.03386.x. PMid:21507146.

3. Saccani R, Valentini NC, Pereira KRG, Müller AB, Gabbard C. Associations of biological factors and affordances in the home with infant motor development. Pediatr Int. 2013;55(2):197-203. http://dx.doi.org/10.1111/ped.12042. PMid:23279095.

4. Bernardi JR, Ferreira CF, Nunes M, da Silva CH, Bosa VL, Silveira PP, et al. Impact of perinatal different intrauterine environments on child growth and development in the first six months of life - IVAPSA birth cohort: rationale, design, and methods. BMC Pregnancy Childbirth. 2012;12(1):25. http://dx.doi.org/10.1186/1471-2393-12-25. PMid:22471837.

5. Correr MT, Ouro MPC, Caçola PM, Almeida TGA, Santos DCC. Adisponibilidade de brinquedos no ambiente domiciliar representa oportunidades para o desenvolvimento motor de lactentes? Temas Desenv. 2014;20(108):25-9.

6. Defilipo EC, Frônio JS, Teixeira MTB, Leite ICG, Bastos RR, Vieira MT, et al. Opportunities in the home environment for motor development. Rev Saude Publica. 2012;46(4):63341. http://dx.doi.org/10.1590/S0034-89102012000400007. PMid:22715001.

7. Freitas TCB, Gabbard C, Caçola P, Montebelo MIL, Santos DCC. Family socioeconomic status and the provision of motor affordances in the home. Braz J Phys Ther. 2013;17(4):31927. http://dx.doi.org/10.1590/S1413-35552013005000096. PMid:24072221.

8. Almeida TGA, Caçola PM, Gabbard C, Correr MT, Vilela GBV Jr, Santos DCC. Comparações entre o desempenho motor e oportunidades de estimulação motora no ambiente domiciliar de lactentes residentes na região sudeste e norte do Brasil. Fisioter Pesqui. [Epub ahead of print].

9. Caçola, P. Affordances in the Home Environment for Motor Development Infant Scale (3-18 months) [Internet]. Arlington: Priscila Caçola, 2015. [cited 2015 April 10]. Available from: http://www.priscilacacola.com/ahemd-is.html.

10. Miquelote AF, Santos DCC, Caçola PM, Montebelo MIL, Gabbard C. Effect of the home environment on motor and cognitive behavior of infants. Infant Behav Dev. 2012;35(3):329-34. http://dx.doi.org/10.1016/j.infbeh.2012.02.002. PMid:22721733. 


\section{Appendix 1}

\section{Affordances no Ambiente Domiciliar para o Desenvolvimento Motor - Escala Bebê (AHEMD-IS) \\ Inventário (3-18 meses) ${ }^{a}$}

\begin{tabular}{|c|c|}
\hline Código & \\
\hline Data & \\
\hline
\end{tabular}

\section{Prezados Pais ou Responsáveis}

Este questionário foi desenvolvido para avaliar as oportunidades (affordances) que sua residência e família proporcionam ao desenvolvimento motor do seu bebê. Os pais conhecem seu bebê muito bem, portanto, são as melhores pessoas para fornecer esse tipo de informação.

É importante que você preencha cada pergunta o mais corretamente possível, pensando no que existe (por exemplo, brinquedos) ou acontece no ambiente familiar que incentiva seu bebê a se movimentar e a brincar. Esperamos que este questionário ajude você a aprender novas formas de estimular o desenvolvimento do seu bebê.

O questionário é composto por uma parte inicial com questões sobre seu bebê e sua família, seguida de três outras partes, que são: Espaço físico do domicílio, Variedade de estimulação e Brinquedos (de motricidade grossa e de motricidade fina) existentes na sua residência.

\section{Características da Criança}

Nome da criança:

Nome da mãe, pai ou responsável:

\begin{tabular}{|c|c|c|c|c|c|}
\hline $\begin{array}{l}\text { Masc. } \square \\
\text { Fem. } \square\end{array}$ & \multicolumn{3}{|c|}{$\begin{array}{l}\text { Data Nascimento: } \\
\text { Peso ao nascer: }\end{array}-\frac{1}{\text { gramas }}$} & \multicolumn{2}{|c|}{$\begin{array}{l}\text { Prematuro: Sim _ Não } \\
\text { Se possível, idade gestacional: }\end{array}$} \\
\hline $\begin{array}{l}\text { Há quanto tempo o seu } \\
\text { filho (a) frequenta a } \\
\text { creche ou escolinha? }\end{array}$ & $\begin{array}{c}\text { Nunca } \\
\square\end{array}$ & $\begin{array}{c}\text { Menos de } 3 \text { meses } \\
\square\end{array}$ & $\begin{array}{c}3-6 \\
\text { meses } \\
\square\end{array}$ & $\begin{array}{c}7-12 \text { meses } \\
\square\end{array}$ & $\begin{array}{c}\text { Acima de } 12 \text { meses } \\
\square\end{array}$ \\
\hline
\end{tabular}

\section{Características da Família}

\begin{tabular}{|c|c|c|c|c|c|c|}
\hline \multicolumn{4}{|l|}{ Tipo de domicílio? } & Apartamento & $\begin{array}{c}\text { Casa } \\
\square\end{array}$ & $\begin{array}{c}\text { Outro } \\
\square\end{array}$ \\
\hline \multicolumn{2}{|l|}{ Quantos adultos vivem no domicílio? } & $\begin{array}{l}1 \\
\square\end{array}$ & $\begin{array}{l}2 \\
\square\end{array}$ & $\begin{array}{l}3 \\
\square\end{array}$ & $\begin{array}{l}4 \\
\square\end{array}$ & $\begin{array}{c}5 \text { ou mais } \\
\square\end{array}$ \\
\hline \multicolumn{2}{|l|}{ Quantas crianças vivem no domicílio? } & $\begin{array}{l}1 \\
\square\end{array}$ & $\begin{array}{l}2 \\
\square\end{array}$ & $\begin{array}{l}3 \\
\square\end{array}$ & $\begin{array}{l}4 \\
\square\end{array}$ & $\begin{array}{c}5 \text { ou mais } \\
\square\end{array}$ \\
\hline $\begin{array}{l}\text { Quantos quartos de dormir há no } \\
\text { domicílio? (não conte banheiros, nem } \\
\text { salas ou cozinha). }\end{array}$ & $\begin{array}{l}0 \\
\square\end{array}$ & $\begin{array}{l}1 \\
\square\end{array}$ & $\begin{array}{l}2 \\
\square\end{array}$ & $\begin{array}{l}3 \\
\square\end{array}$ & $\begin{array}{l}4 \\
\square\end{array}$ & $\begin{array}{c}5 \text { ou mais } \\
\square\end{array}$ \\
\hline $\begin{array}{l}\text { Há quanto tempo sua família vive neste } \\
\text { domicílio? }\end{array}$ & \multicolumn{2}{|c|}{ Menos de 3 meses } & $\begin{array}{c}\text { 3-6 meses } \\
\square\end{array}$ & $\begin{array}{c}\text { 7-12 meses } \\
\square\end{array}$ & \multicolumn{2}{|c|}{ Acima de 12 meses } \\
\hline Qual o grau de escolaridade do pai? & $\begin{array}{r}\text { Sen } \\
\text { fundam }\end{array}$ & $\begin{array}{l}\text { o ou } \\
\text { ompleto }\end{array}$ & $\begin{array}{c}\text { Fundamental } \\
\text { completo } \\
\square\end{array}$ & $\begin{array}{l}\text { Médio } \\
\text { completo } \\
\square\end{array}$ & $\begin{array}{c}\text { Superior } \\
\text { completo } \\
\square\end{array}$ & $\begin{array}{c}\text { Mestrado ou } \\
\text { Doutorado } \\
\square\end{array}$ \\
\hline Qual o grau de escolaridade da mãe? & & & $\square$ & $\square$ & $\square$ & $\square$ \\
\hline
\end{tabular}

${ }^{a}$ C Esse questionário foi desenvolvido pelo Developmental Motor Cognition Lab - University of Texas at Arlington (USA), Motor Development Lab - Texas A\&M University (USA) e Laboratório de Pesquisa em Desenvolvimento Neuromotor - Universidade Metodista de Piracicaba (Brasil). Todos os direitos reservados. 
Instruções: Leia cuidadosamente cada questão e marque o quadrado (alternativa) que melhor representa sua resposta:

\section{ESPAÇO FÍSICO DA RESIDÊNCIA}

\begin{tabular}{|l|l|c|}
\hline \multicolumn{2}{|l|}{$\begin{array}{l}\text { A sua residência tem algum ESPAÇO EXTERNO, seguro, adequado e amplo para seu bebê brincar e } \\
\text { se movimentar livremente (se arrastar, engatinhar ou andar)? (área na frente, área no fundo, quintal, } \\
\text { jardim, terraço, etc.). } \\
\begin{array}{l}\text { Obs. Caso more em apartamento, considere como espaço externo o parquinho ou área de lazer do seu } \\
\text { prédio ou condomínio. }\end{array}\end{array}$} & NIM \\
\hline
\end{tabular}

ATENÇÃO: Se você respondeu SIM, continue com as próximas questões. Se você respondeu NÃO, passe para a questão número 6 .

\begin{tabular}{|c|l|c|}
\hline No espaço EXTERNO da sua residência existe ... & SIM & NÃO \\
\hline 2. & $\begin{array}{l}\text { Mais do que um tipo de piso ou solo na área externa? (grama, cimento, piso frio ou ladrilho, areia, } \\
\text { madeira, etc.). }\end{array}$ & $\square$ \\
\hline 3. & Uma ou mais superfícies inclinadas? (rampas no quintal, escorregador para bebês). & $\square$ \\
\hline $\begin{array}{l}\text { Algum suporte ou mobília que seja seguro, na área externa, onde seu bebê possa se apoiar para se } \\
\text { levantar e/ou andar? (portão/grades, mesa baixa de jardim, bancos/cadeiras, muros baixos/mureta, } \\
\text { etc.). }\end{array}$ & $\square$ \\
\hline $\begin{array}{l}\text { Degraus ou escada na área externa? (degrau na porta da frente ou dos fundos, degraus em um } \\
\text { escorregador para bebês). } \\
\text { Obs. Escadas oferecem risco ao bebê. Use portões de segurança no topo e no pé da escada. }\end{array}$ & $\square$ \\
\hline No espaço INTERNO (dentro da sua residência) existe ... & $\square$ \\
\hline 6. $\begin{array}{l}\text { Mais do que um tipo de piso no espaço interno? (cimento, piso frio ou ladrilho, carpete, carpete de } \\
\text { madeira, madeira, etc.). }\end{array}$ & $\square$ \\
\hline 7. $\begin{array}{l}\text { Degraus ou escada no espaço interno? } \\
\text { Obs. Escadas oferecem risco ao bebê. Use portões de segurança no topo e no pé da escada. }\end{array}$ & NÃO \\
\hline
\end{tabular}

\section{VARIEDADE DE ESTIMULAÇÃO}

As questões seguintes referem-se SOMENTE ao tempo em que o seu bebê está em casa.

Obs. Não considerar o que ocorre na creche ou escolinha.

8. O meu/nosso bebê brinca regularmente (pelo menos duas vezes por semana) com outras crianças.

9. Eu/nós, regularmente (pelo menos duas vezes por semana), fazemos brincadeiras que encorajam nosso bebê a aprender sobre as partes do corpo. (Por exemplo, onde está sua mão?).

\begin{tabular}{|c|c|c|}
\hline SIM & NÃO \\
\hline & $\square$ & $\square$ \\
\hline & $\square$ \\
\hline
\end{tabular}

${ }^{a}(\mathrm{C}$ Esse questionário foi desenvolvido pelo Developmental Motor Cognition Lab - University of Texas at Arlington (USA), Motor Development Lab - Texas A\&M University (USA) e Laboratório de Pesquisa em Desenvolvimento Neuromotor - Universidade Metodista de Piracicaba (Brasil). Todos os direitos reservados. 


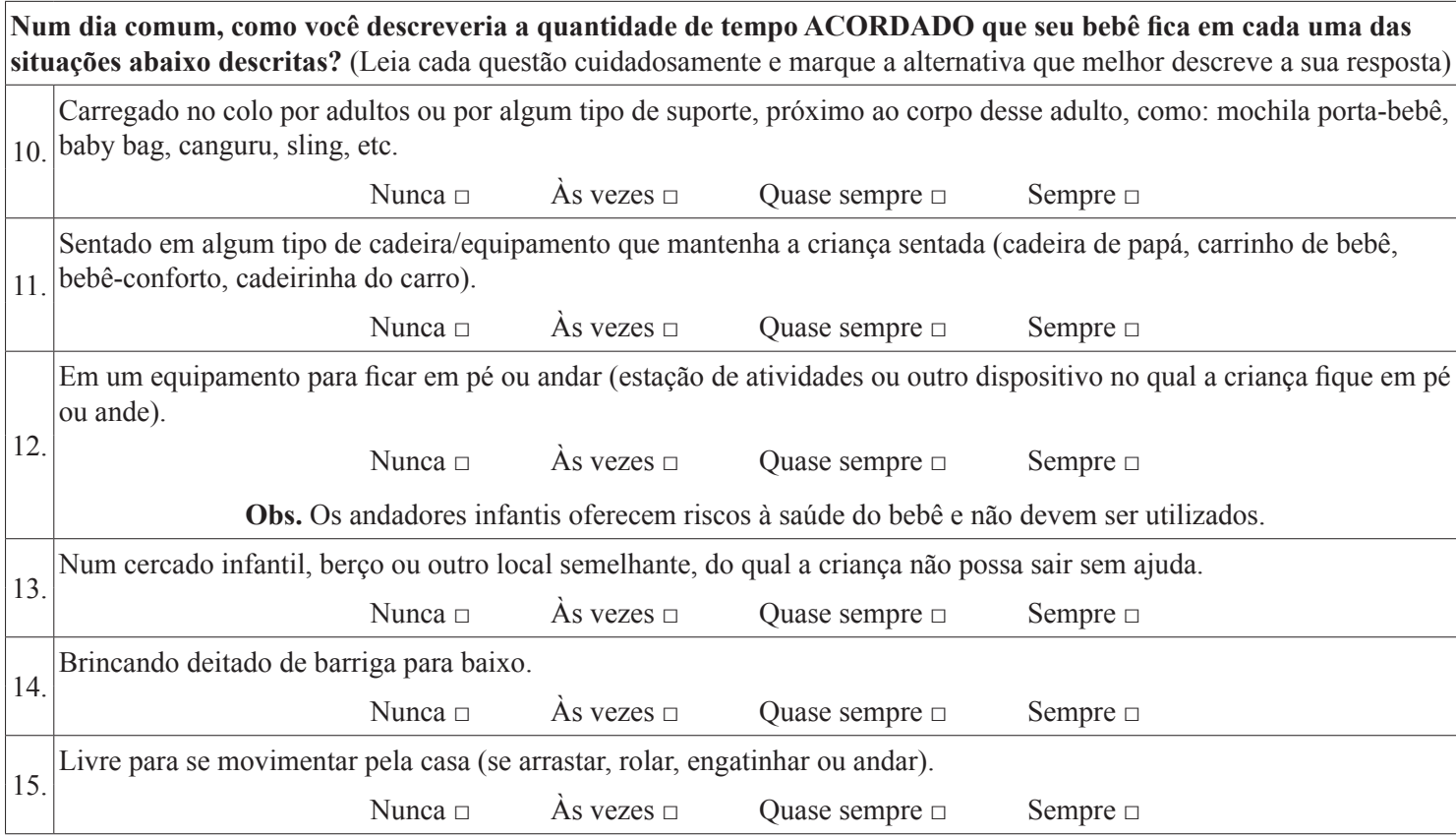

\section{BRINQUEDOS (Motricidade Grossa e Motricidade Fina)}

Instruções: Para cada grupo de brinquedo listado abaixo, marque o número de brinquedos iguais ou SEMELHANTES que você utiliza (em sua casa) para brincar com seu bebê.

Por favor, leia cuidadosamente a descrição geral de cada grupo antes de decidir se você tem em casa esse tipo de brinquedo. AS FIGURAS SÃO APENAS EXEMPLOS para ajudar você a entender melhor a descrição. Você NÃO precisa ter exatamente os mesmos brinquedos que estão neste questionário para contá-los no grupo. BRINQUEDOS SEMELHANTES do mesmo TIPO devem ser contados.

\section{BRINQUEDOS - MOTRICIDADE GROSSA (questões 16 a 21)}

16. Brinquedos suspensos acima ou ao lado do bebê, móbiles e/ou enfeites de berço.

Exemplos são:
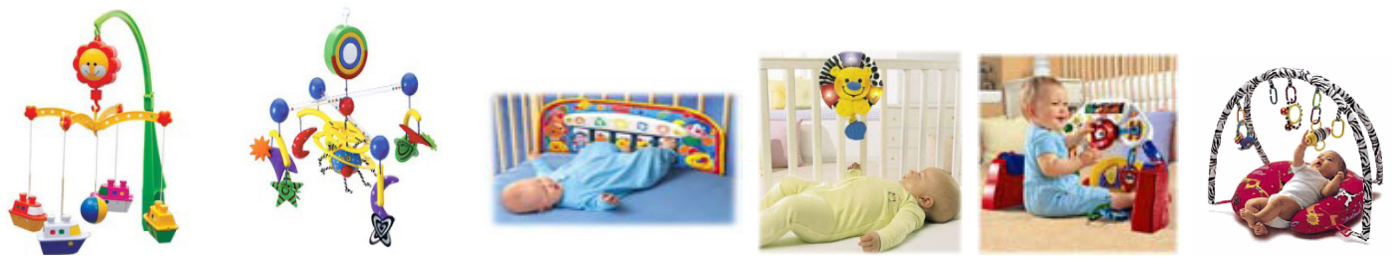

Quantos destes brinquedos você utiliza para brincar com seu bebê em casa?

Nenhum $\square \quad$ Um - dois $\square \quad$ Três ou mais $\square$

${ }^{a}$ C Esse questionário foi desenvolvido pelo Developmental Motor Cognition Lab - University of Texas at Arlington (USA), Motor Development Lab - Texas A\&M University (USA) e Laboratório de Pesquisa em Desenvolvimento Neuromotor - Universidade Metodista de Piracicaba (Brasil). Todos os direitos reservados. 
17. Bonecos de pelúcia (musicais ou não), brinquedos emborrachados, de tecido ou outros materiais macios, de brincar na água (flutuantes, esponjas).

Exemplos são:
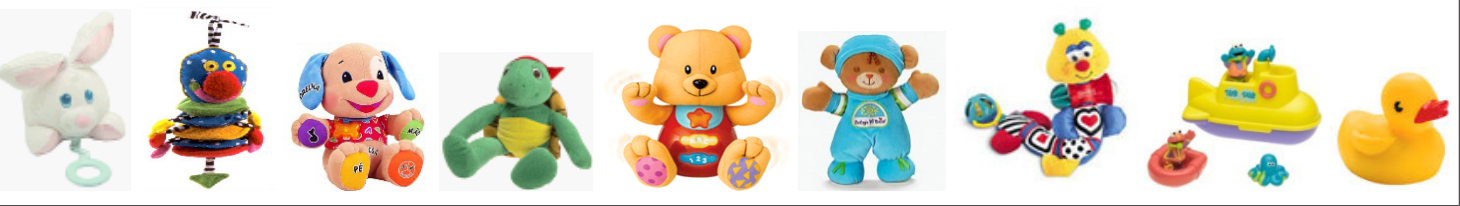

Quantos destes brinquedos você utiliza para brincar com seu bebê em casa?

Nenhum $\square \quad$ Um - dois $\square \quad$ Três ou mais $\square$

18. Cadeirinhas de balanços para bebês, estação de atividades (o bebê fica em pé dentro da estação brincando), balanços para bebês.

Exemplos são:
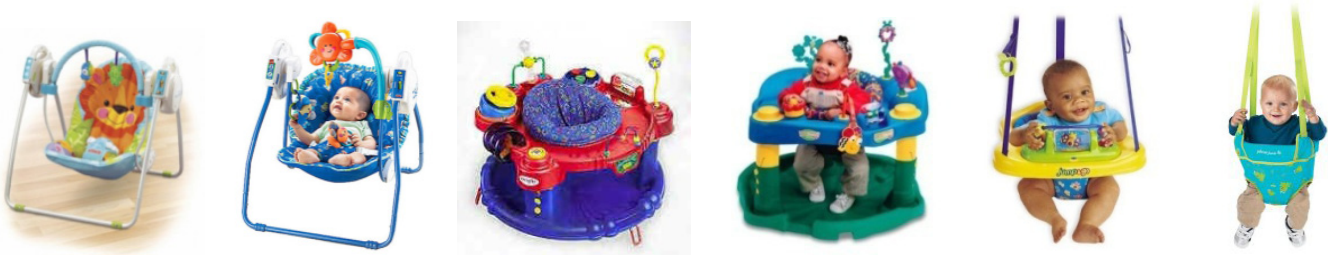

Quantos destes brinquedos você utiliza para brincar com seu bebê em casa?

Nenhum $\square \quad$ Um - dois $\square \quad$ Três ou mais $\square$

19. Bolas de diferentes tamanhos, texturas, cores e formas.

Exemplos são:
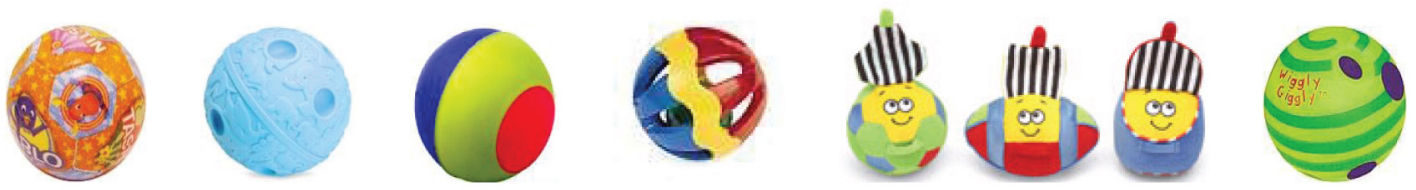

Quantos destes brinquedos você utiliza para brincar com seu bebê em casa?

Nenhum $\square \quad$ Um - dois $\square \quad$ Três ou mais $\square$

20. Materiais que estimulem a criança a se arrastar, rolar, engatinhar ou até se levantar (colchonetes, tapete emborrachado, plataformas macias, etc.).

Exemplos são:
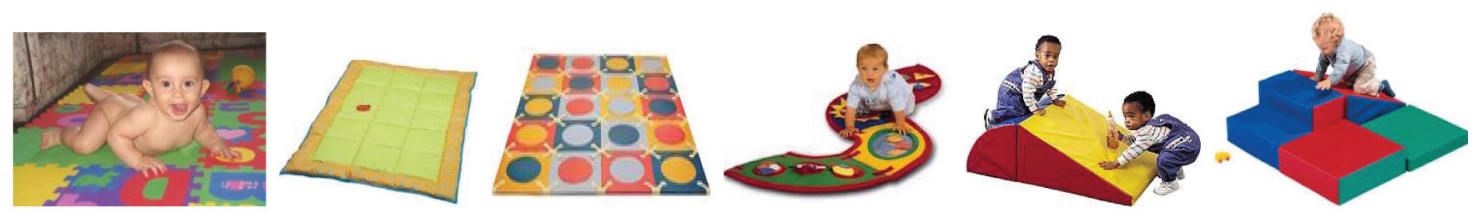

Quantos destes brinquedos você utiliza para brincar com seu bebê em casa?

Nenhum $\square \quad$ Um - dois $\square \quad$ Três ou mais $\square$

${ }^{a}(\mathbb{C}$ Esse questionário foi desenvolvido pelo Developmental Motor Cognition Lab - University of Texas at Arlington (USA), Motor Development Lab - Texas A\&M University (USA) e Laboratório de Pesquisa em Desenvolvimento Neuromotor - Universidade Metodista de Piracicaba (Brasil). Todos os direitos reservados. 
21. Materiais musicais: instrumentos, caixas de música e brinquedos que emitem sons e melodias em resposta às ações da criança (chacoalhar, pressionar, puxar, etc.).

Exemplos são:
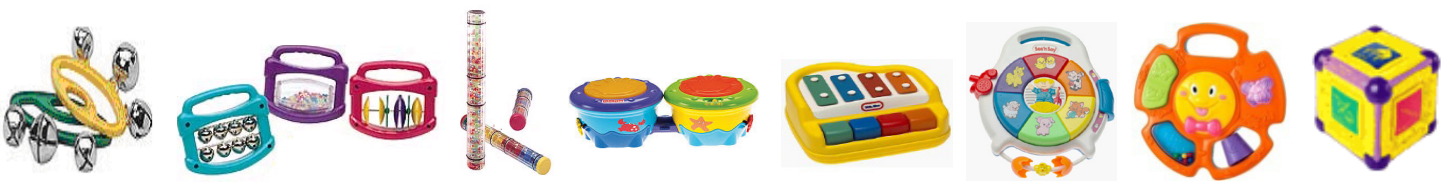

Quantos destes brinquedos você utiliza para brincar com seu bebê em casa?

Nenhum $\square$

Um - dois $\square$

Três ou mais $\square$

\section{BRINQUEDOS - MOTRICIDADE FINA (questões 22 a 26)}

22. Brinquedos manipuláveis: chocalhos, mordedores, brinquedos com diferentes texturas e/ou com espelho.

Exemplos são:
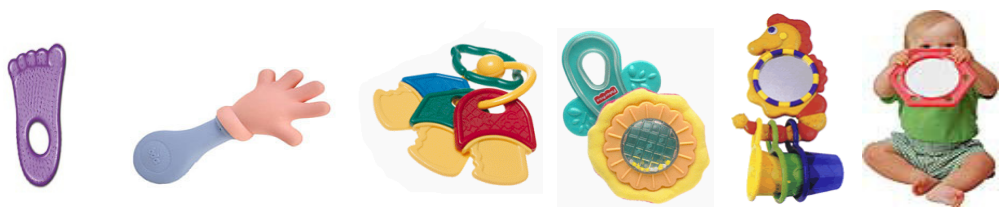

Quantos destes brinquedos você utiliza para brincar com seu bebê em casa?

Nenhum $\square \quad$ Um - dois $\square \quad$ Três ou mais $\square$

\section{Carros, trens, animais ou outros brinquedos que possam ser puxados ou empurrados.}

Exemplos são:
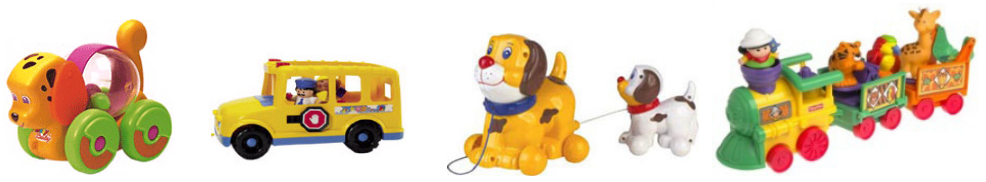

Quantos destes brinquedos você utiliza para brincar com seu bebê em casa?
Nenhum $\square$
Um - dois $\square$
Três ou mais $\square$

\section{Brinquedos de apertar (pressionar), bater e acionar, peões, gira-giras.}

Exemplos são:
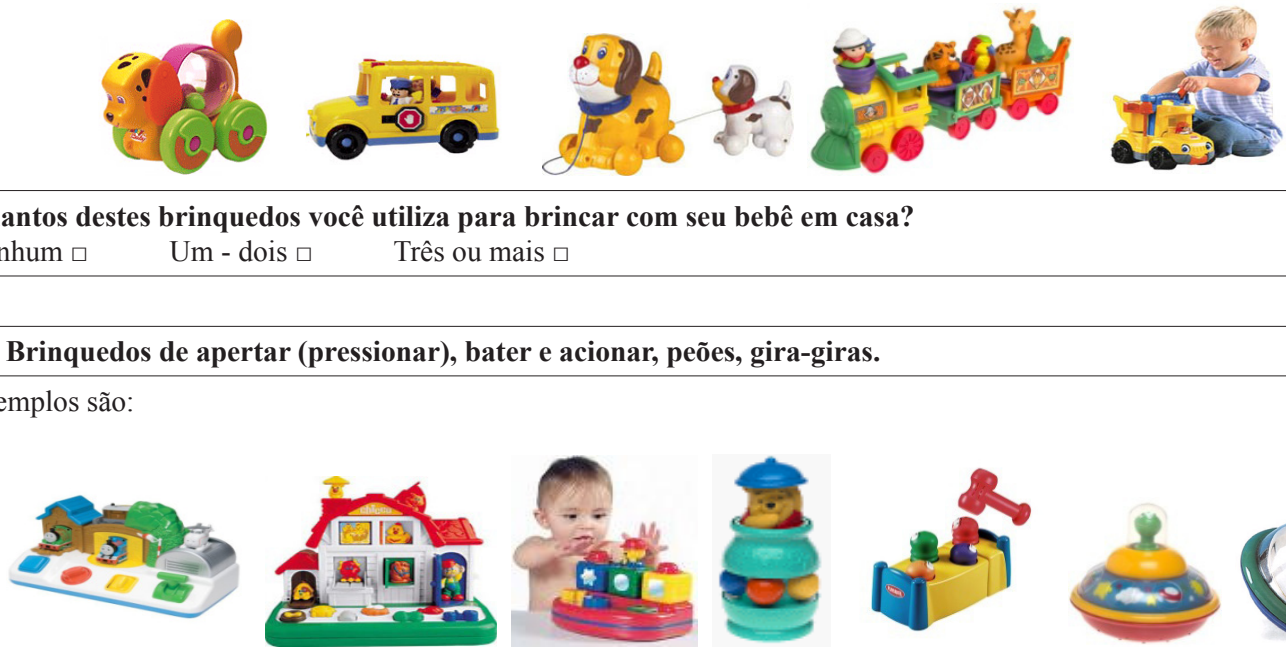

Quantos destes brinquedos você utiliza para brincar com seu bebê em casa?

Nenhum $\square \quad$ Um - dois $\square \quad$ Três ou mais $\square$ 


\section{Blocos de montar (plástico, espuma, tecido, madeira, borracha)}

Exemplos são:
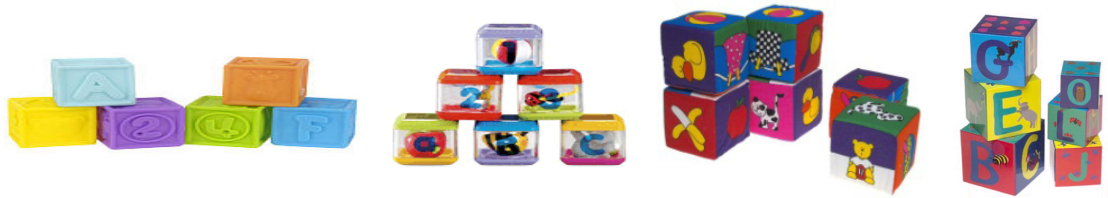

Quantos destes brinquedos você utiliza para brincar com seu bebê em casa?

Nenhum $\square \quad$ Um - dois $\square \quad$ Três ou mais $\square$

\section{Livros para bebês (tecido, papel cartão ou plástico).}

Exemplos são:
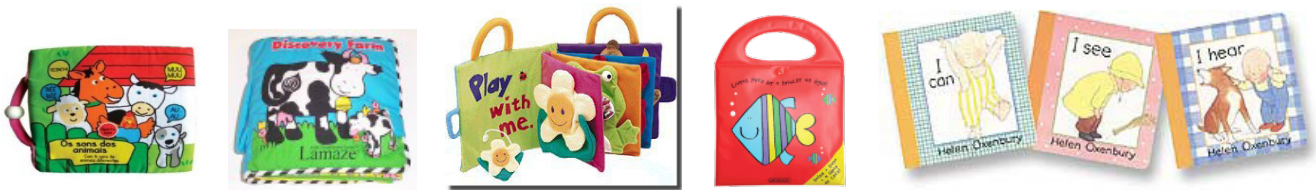

Quantos destes brinquedos você utiliza para ler e brincar com seu bebê em casa?

Nenhum $\square \quad$ Um - dois $\square \quad$ Três ou mais $\square$

ATENÇÃO:

- Se o seu bebê tem entre 03 e 11 MESES de idade PARE aqui de responder o questionário.

- Os brinquedos a seguir NÃO são recomendados para crianças com MENOS de 12 meses.

- Se o seu bebê tem 12 MESES ou mais de idade CONTINUE respondendo o questionário.

\section{BRINQUEDOS - MOTRICIDADE GROSSA (questões 27 a 29)}

27. Objetos ou brinquedos que estimulam a criança a se levantar e a caminhar com apoio (brinquedos de empurrar e puxar).

Exemplos são:
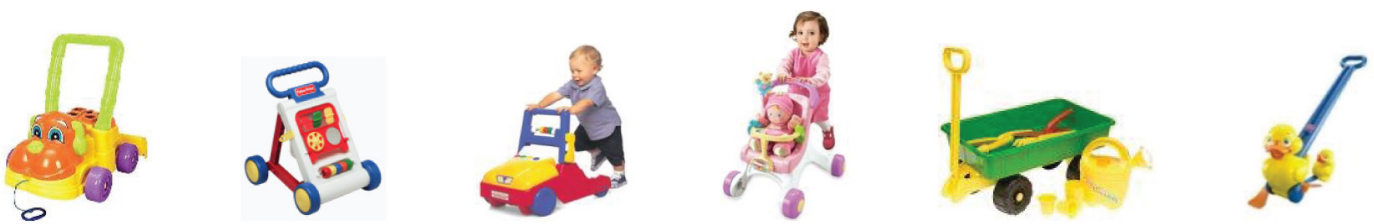

Quantos destes brinquedos você utiliza para brincar com seu bebê em casa?

Nenhum $\square \quad$ Um - dois $\square \quad$ Três ou mais $\square$

28. Mesinhas de atividades onde o bebê possa brincar em pé (plástico, madeira, etc.).

Exemplos são:
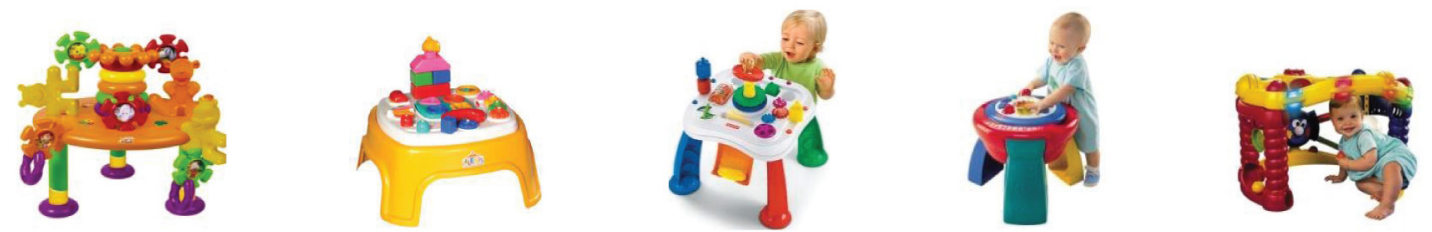

Quantos destes brinquedos você utiliza para brincar com seu bebê em casa?

Nenhum $\square \quad$ Um - dois $\square \quad$ Três ou mais $\square$

${ }^{a_{C}}$ Esse questionário foi desenvolvido pelo Developmental Motor Cognition Lab - University of Texas at Arlington (USA), Motor Development Lab - Texas A\&M University (USA) e Laboratório de Pesquisa em Desenvolvimento Neuromotor - Universidade Metodista de Piracicaba (Brasil). Todos os direitos reservados. 


\section{Balanços ao ar livre para bebês, cavalos de balanço, triciclos para bebês.}

Exemplos são:
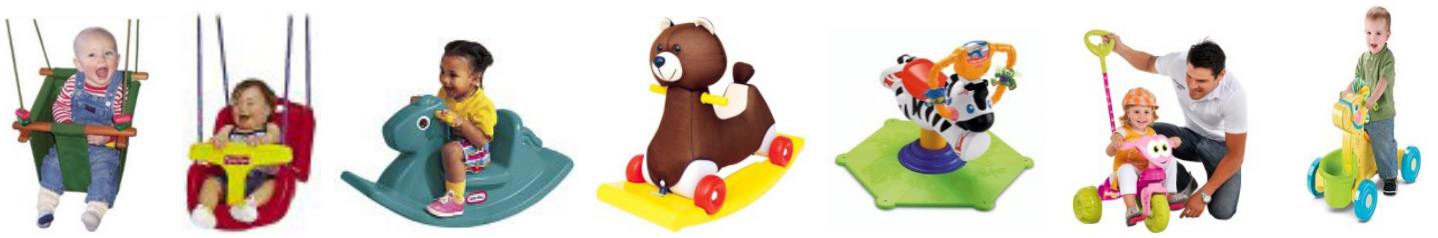

Quantos destes brinquedos você utiliza para brincar com seu bebê em casa?

Nenhum $\square \quad$ Um - dois $\square \quad$ Três ou mais $\square$

\section{BRINQUEDOS - MOTRICIDADE FINA (questões 30 a 35)}

\section{Brinquedos educativos para encaixar formas variadas.}

Exemplos são:
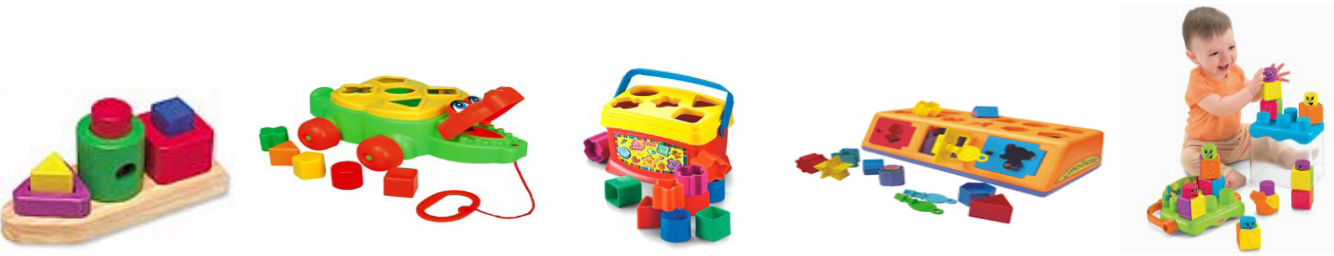

Quantos destes brinquedos que você utiliza para brincar com seu bebê em casa?

Nenhum $\square \quad$ Um - dois $\square \quad$ Três ou mais $\square$

\section{Fantoches e marionetes macios.}

Exemplos são:
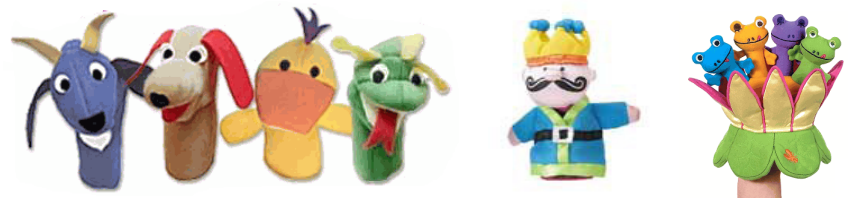

Quantos destes brinquedos você utiliza para brincar com seu bebê em casa?
Nenhum $\square$
Um - dois $\square$
Três ou mais $\square$

\section{Bonecos(as) e outros personagens com acessórios (mamadeira, roupas, capacete, mobiliário, etc.).}

Exemplos são:
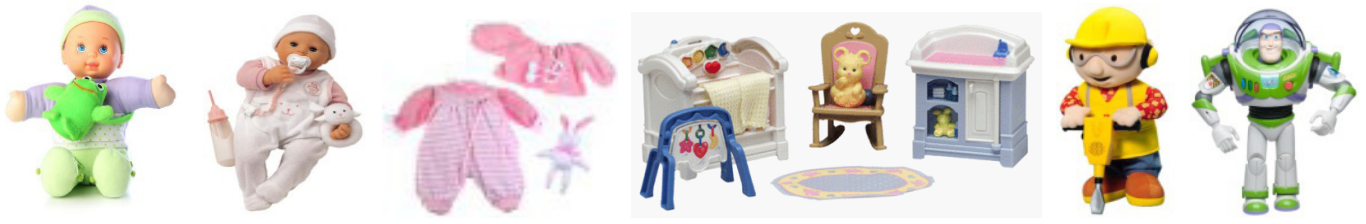

Quantos destes brinquedos você utiliza para brincar com seu bebê em casa?

Nenhum $\square \quad$ Um - dois $\square \quad$ Três ou mais $\square$

${ }^{a} \mathbb{C}$ Esse questionário foi desenvolvido pelo Developmental Motor Cognition Lab - University of Texas at Arlington (USA), Motor Development Lab - Texas A\&M University (USA) e Laboratório de Pesquisa em Desenvolvimento Neuromotor - Universidade Metodista de Piracicaba (Brasil). Todos os direitos reservados. 


\section{Brinquedos que imitam objetos existentes na casa: telefones, ferramentas, utensílios de cozinha, etc.}

Exemplos são:
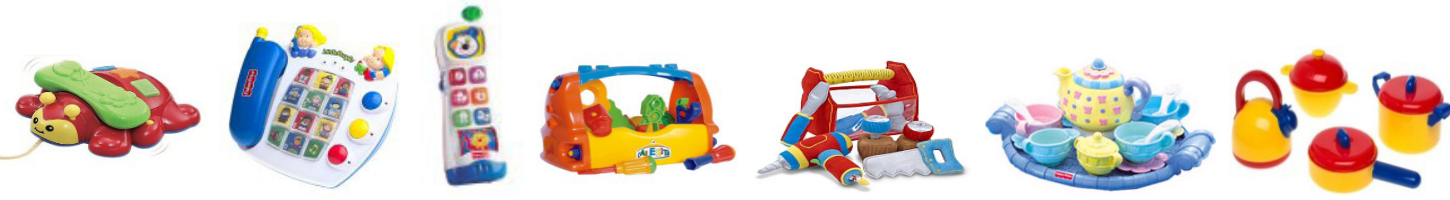

Quantos destes brinquedos você utiliza para brincar com seu bebê em casa?

Nenhum $\square \quad$ Um - dois $\square \quad$ Três ou mais $\square$

\section{Brinquedos de empilhar.}

Exemplos são:
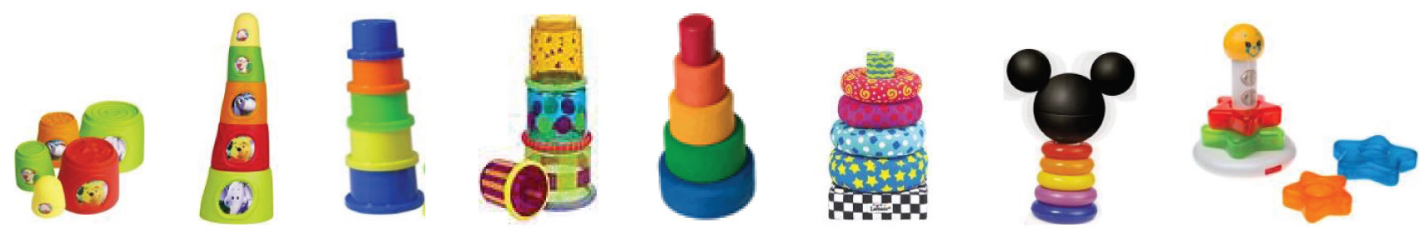

Quantos destes brinquedos você utiliza para brincar com seu bebê em casa?

Nenhum $\square \quad$ Um - dois $\square \quad$ Três ou mais $\square$

\section{Quebra-cabeças para bebês (2-6 peças).}

Exemplos são:
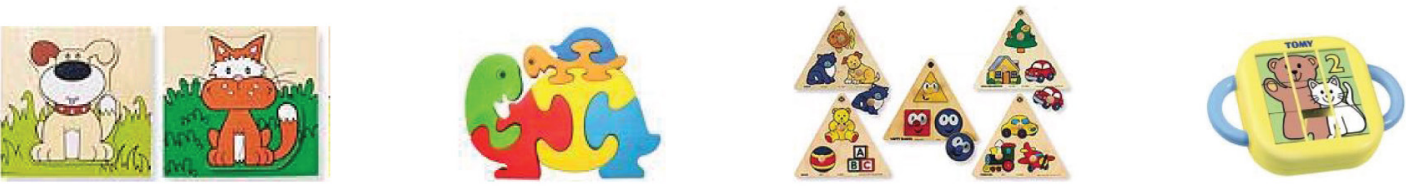

Quantos destes brinquedos você utiliza para brincar com seu bebê em casa?

Nenhum $\square \quad$ Um - dois $\square \quad$ Três ou mais $\square$

\section{Informações adicionais:}




\section{Appendix 2}

\section{AFFORDANCES NO AMBIENTE DOMICILIAR PARA 0 DESENVOLVIMENTO MOTOR ESCALA BEBÊ (AHEMD-IS) ${ }^{a}$}

\section{Folha de pontuação - Bebês 3 a 11 meses}

1. Indique o valor dos pontos para a resposta de cada questão (Tabela 1.1)

2. Some os pontos para cada dimensão

3. Some o total de pontos para as quatro dimensões

Tabela 1.1. Registro de pontos para 3 a 11 meses.

\begin{tabular}{|c|c|c|}
\hline Dimensão & Questão & $\operatorname{Sim}=1$, Não $=0$ \\
\hline \multirow{7}{*}{ Espaço Físico } & 1 & \\
\hline & 2 & \\
\hline & 3 & \\
\hline & 4 & \\
\hline & 5 & \\
\hline & 6 & \\
\hline & 7 & \\
\hline TOTAL & Somar os pontos & \\
\hline \multirow{11}{*}{ Variedade de Estimulação } & Questão & $\operatorname{Sim}=1$, Não $=0$ \\
\hline & 8 & \\
\hline & 9 & \\
\hline & & Nunca $=3 /$ Às vezes $=2 /$ Quase sempre $=1 /$ Sempre $=0$ \\
\hline & 10 & \\
\hline & 11 & \\
\hline & 12 & \\
\hline & 13 & \\
\hline & & Nunca $=0 /$ Às vezes $=1 /$ Quase sempre $=2 /$ Sempre $=3$ \\
\hline & 14 & \\
\hline & 15 & \\
\hline TOTAL & Somar os pontos & \\
\hline \multirow{7}{*}{ Brinquedos de Motricidade Grossa } & Questão & Nenhum $=0 /$ Um - dois $=1 /$ Três ou mais $=2$ \\
\hline & 16 & \\
\hline & 17 & \\
\hline & 18 & \\
\hline & 19 & \\
\hline & 20 & \\
\hline & 21 & \\
\hline TOTAL & Somar os pontos & \\
\hline
\end{tabular}

${ }^{a}$ C Esse questionário foi desenvolvido pelo Developmental Motor Cognition Lab - University of Texas at Arlington (USA), Motor Development Lab - Texas A\&M University (USA) e Laboratório de Pesquisa em Desenvolvimento Neuromotor - Universidade Metodista de Piracicaba (Brasil). Todos os direitos reservados. 
Tabela 1.1. Continuação...

\begin{tabular}{|l|c|l|}
\hline \multirow{4}{*}{ Dimensão } & & \\
\hline \multirow{4}{*}{ Brinquedos de Motricidade Fina } & Questão & Nenhum $=0 /$ Um - dois $=1$ / Três ou mais $=2$ \\
\cline { 2 - 3 } & 22 & \\
\cline { 2 - 3 } & 23 & \\
\cline { 2 - 3 } & 24 & \\
\cline { 2 - 3 } & 25 & \\
\hline & 26 & \\
\hline TOTAL & Somar os pontos & \\
\hline Pontuação TOTAL - 4 dimensões & Somar os pontos & \\
\hline
\end{tabular}




\section{AFFORDANCES NO AMBIENTE DOMICILIAR PARA O DESENVOLVIMENTO MOTOR ESCALA BEBÊ (AHEMD-IS)}

\section{Folha de pontuação - Bebês 3 a 11 meses}

4. Copie o total de pontos de cada dimensão e a pontuação total na tabela abaixo (Tabela 1.2)

5. Selecione a categoria descritiva para cada dimensão e a pontuação total (Tabela 1.3)

Tabela 1.2. Registro do total de pontos e categoria descritiva para 3 a 11 meses.

\begin{tabular}{|l|l|l|}
\hline \multicolumn{1}{|c|}{ Dimensões } & Pontos & Categoria Descritiva \\
\hline 1. Espaço Físico & & \\
\hline 2. Variedade de Estimulação & & \\
\hline 3. Brinquedos de Motricidade Grossa & & \\
\hline 4. Brinquedos de Motricidade Fina & & \\
\hline Pontuação Total & & \\
\hline
\end{tabular}

Tabela 1.3. Convertendo pontuações em categorias descritivas do ambiente (por dimensão e pontuação total) para 3 a 11 meses.

\begin{tabular}{|c|c|c|c|c|c|c|}
\hline IDADE & $\begin{array}{l}\text { CATEGORIAS } \\
\text { DESCRITIVAS }\end{array}$ & $\begin{array}{l}\text { ESPAÇO } \\
\text { FÍSICO }\end{array}$ & $\begin{array}{l}\text { VARIEDADE DE } \\
\text { ESTIMULAÇÃO }\end{array}$ & \begin{tabular}{|c} 
BRINQUEDOS DF \\
MOTRICIDADE \\
FINA
\end{tabular} & $\begin{array}{c}\text { BRINQUEDOS DE } \\
\text { MOTRICIDADE } \\
\text { GROSSA } \\
\end{array}$ & $\begin{array}{l}\text { PONTUAÇÃO } \\
\text { TOTAL }\end{array}$ \\
\hline \multirow{4}{*}{$\begin{array}{c}3-11 \\
\text { MESES }\end{array}$} & $\begin{array}{l}\text { MENOS QUE } \\
\text { ADEQUADO }\end{array}$ & $0-1$ & $0-9$ & $0-2$ & $0-3$ & $0-18$ \\
\hline & $\begin{array}{l}\text { MODERADAMENTE } \\
\text { ADEQUADO }\end{array}$ & $2-3$ & $10-11$ & 3 & $4-5$ & $19-23$ \\
\hline & ADEQUADO & $4-5$ & $12-13$ & $4-5$ & $6-7$ & $24-27$ \\
\hline & EXCELENTE & $6-7$ & $14-20$ & $6-10$ & $8-12$ & $28-49$ \\
\hline
\end{tabular}

\section{Categorias Descritivas:}

Menos que adequado: As oportunidades (affordances) que o ambiente oferece ao desenvolvimento motor do bebê estão faltando (são poucas) ou precisam ser melhoradas. Sugestão: A família deve procurar adicionar ou melhorar certas dimensões do ambiente domiciliar, oferecendo variedade de oportunidades que podem beneficiar o desenvolvimento motor do seu bebê.

Moderadamente adequado: $\mathrm{O}$ ambiente oferece algumas oportunidades (affordances) para o desenvolvimento motor, porém, estas poderiam ser melhoradas. Sugestão: Acrescentar mais oportunidades que podem ajudar a estimular o desenvolvimento motor do seu bebê.

Adequado: $\mathrm{O}$ ambiente mostra suficiente quantidade e qualidade de oportunidades (affordances). Sugestão: Continuar usando e buscando formas diferentes de explorar as oportunidades que o lar pode proporcionar ao desenvolvimento motor do seu bebê.

Excelente: $\mathrm{O}$ ambiente domiciliar oferece ampla quantidade e variedade de oportunidades (affordances). Sugestão: Continuar usando e procurando formas diferentes de encorajar o movimento e brincadeiras com o seu bebê.

Alguns exemplos de como usar o AHEMD - Escala Bebê para melhorar o ambiente do lar para o desenvolvimento motor:

Ao final da avaliação, você terá 5 fatores para interpretar: 4 dimensões (Espaço Físico, Variedade de Estimulação, Brinquedos de Motricidade Fina, Brinquedos de Motricidade Grossa) e a Pontuação Total.

${ }^{a} \mathbb{C}$ Esse questionário foi desenvolvido pelo Developmental Motor Cognition Lab - University of Texas at Arlington (USA), Motor Development Lab - Texas A\&M University (USA) e Laboratório de Pesquisa em Desenvolvimento Neuromotor - Universidade Metodista de Piracicaba (Brasil). Todos os direitos reservados. 
Os resultados das categorias descritivas vão ajudar no entendimento de como cada dimensão se comporta em relação à pontuação total do ambiente. Por exemplo, uma ou duas dimensões com pontuação baixa podem contribuir para uma pontuação total baixa. Se esse for o caso, é importante focar nestas dimensões para melhorar as oportunidades para o desenvolvimento motor do bebê.

Além da análise por dimensão, é muito importante verificar os itens específicos dentro de cada dimensão para determinar quais são os aspectos do lar que devem ser adicionados ou modificados para melhorar oportunidades de ação para o bebê.

Exemplos para melhorar cada dimensão são:

- Espaço Físico: Se há uma falta de tipos de piso/solo diferenciados (questão 2) ou de degraus/escadas (questões 5 e 7) no espaço interior e exterior, leve o bebê para lugares fora da casa que possam oferecer tais oportunidades;

- Variedade de Estimulação: A falta de brincadeiras que encorajam aprender sobre as partes do corpo (questão 9) ou a falta de costume de colocar o bebê para brincar deitado, de barriga para baixo (questão 14), podem ser facilmente incluídos na rotina diária do bebê;

- Brinquedos de Motricidade Fina: A falta de brinquedos musicais (questão 21) ou de livros para bebês (questão 26) pode direcionar os pais na próxima vez que forem comprar um novo brinquedo;

- Brinquedos de Motricidade Grossa: A falta de balanços para bebês (questão 29) pode ser compensada, levando o bebê a lugares fora de casa que têm esse tipo de brinquedo e expor a criança a tais oportunidades. A falta de um tapete emborrachado (questão 20) pode ser compensada por uma superfície confortável (um colchonete ou acolchoado no chão) que permita ao bebê ser mais ativo (rolar, engatinhar, etc.).

Observações: 


\section{AFFORDANCES NO AMBIENTE DOMICILIAR PARA 0 DESENVOLVIMENTO MOTOR \\ ESCALA BEBÊ (AHEMD-IS)}

\section{Folha de pontuação - Bebês 12 a 18 meses}

1. Indique o valor dos pontos para a resposta de cada questão (Tabela 2.1)

2. Some os pontos para cada dimensão

3. Some o total de pontos para as quatro dimensões

Tabela 2.1. Registro de pontos para 12 a 18 meses.

\begin{tabular}{|c|c|c|}
\hline Dimensão & Questão & $\operatorname{Sim}=1$, Não $=0$ \\
\hline \multirow{7}{*}{ Espaço Físico } & 1 & \\
\hline & 2 & \\
\hline & 3 & \\
\hline & 4 & \\
\hline & 5 & \\
\hline & 6 & \\
\hline & 7 & \\
\hline TOTAL & Somar os pontos & \\
\hline \multirow{11}{*}{ Variedade de Estimulação } & Questão & $\operatorname{Sim}=1$, Não $=0$ \\
\hline & 8 & \\
\hline & 9 & \\
\hline & & Nunca $=3 /$ Às vezes $=2 /$ Quase sempre $=1 /$ Sempre $=0$ \\
\hline & 10 & \\
\hline & 11 & \\
\hline & 12 & \\
\hline & 13 & \\
\hline & & Nunca $=0 /$ Às vezes $=1 /$ Quase sempre $=2 /$ Sempre $=3$ \\
\hline & 14 & \\
\hline & 15 & \\
\hline TOTAL & Somar os pontos & \\
\hline \multirow{10}{*}{ Brinquedos de Motricidade Grossa } & Questão & Nenhum $=0 /$ Um - dois $=1 /$ Três ou mais $=2$ \\
\hline & 16 & \\
\hline & 17 & \\
\hline & 18 & \\
\hline & 19 & \\
\hline & 20 & \\
\hline & 21 & \\
\hline & 27 & \\
\hline & 28 & \\
\hline & 29 & \\
\hline TOTAL & Somar os pontos & \\
\hline
\end{tabular}

${ }^{a}$ C Esse questionário foi desenvolvido pelo Developmental Motor Cognition Lab - University of Texas at Arlington (USA), Motor Development Lab - Texas A\&M University (USA) e Laboratório de Pesquisa em Desenvolvimento Neuromotor - Universidade Metodista de Piracicaba (Brasil). Todos os direitos reservados. 
Tabela 2.1. Continuação...

\begin{tabular}{|c|c|c|}
\hline Dimensão & & \\
\hline \multirow{12}{*}{ Brinquedos de Motricidade Fina } & Questão & Nenhum $=0 /$ Um - dois $=1 /$ Três ou mais $=2$ \\
\hline & 22 & \\
\hline & 23 & \\
\hline & 24 & \\
\hline & 25 & \\
\hline & 26 & \\
\hline & 30 & \\
\hline & 31 & \\
\hline & 32 & \\
\hline & 33 & \\
\hline & 34 & \\
\hline & 35 & \\
\hline TOTAL & Somar os pontos & \\
\hline Pontuação TOTAL - 4 dimensões & Somar os pontos & \\
\hline
\end{tabular}




\title{
AFFORDANCES NO AMBIENTE DOMICILIAR PARA 0 DESENVOLVIMENTO MOTOR
}

\author{
ESCALA BEBE (AHEMD-IS)
}

\section{Folha de pontuação - Bebês 12 a 18 meses}

4. Copie o total de pontos de cada dimensão e a pontuação total na tabela abaixo (Tabela 2.2)

5. Selecione a categoria descritiva para cada dimensão e a pontuação total (Tabela 2.3)

Tabela 2.2. Registro do total de pontos e categoria descritiva para 3 a 11 meses.

\begin{tabular}{|l|l|l|}
\hline \multicolumn{1}{|c|}{ Dimensões } & Pontos & Categoria Descritiva \\
\hline 1. Espaço Físico & & \\
\hline 2. Variedade de Estimulação & & \\
\hline 3. Brinquedos de Motricidade Grossa & & \\
\hline 4. Brinquedos de Motricidade Fina & & \\
\hline Pontuação Total & & \\
\hline
\end{tabular}

Tabela 2.3. Convertendo pontuações em categorias descritivas do ambiente (por dimensão e pontuação total) para 12 a 18 meses.

\begin{tabular}{|c|c|c|c|c|c|c|}
\hline IDADE & $\begin{array}{l}\text { CATEGORIAS } \\
\text { DESCRITIVAS }\end{array}$ & $\begin{array}{l}\text { ESPAÇO } \\
\text { FÍSICO }\end{array}$ & $\begin{array}{l}\text { VARIEDADE DE } \\
\text { ESTIMULAÇÃO }\end{array}$ & \begin{tabular}{|c|} 
BRINQUEDOS DE \\
MOTRICIDADE \\
FINA
\end{tabular} & \begin{tabular}{|c|} 
BRINQUEDOS DE \\
MOTRICIDADE \\
GROSSA
\end{tabular} & $\begin{array}{l}\text { PONTUAÇÃO } \\
\text { TOTAL }\end{array}$ \\
\hline \multirow{4}{*}{$\begin{array}{c}12-18 \\
\text { MESES }\end{array}$} & $\begin{array}{l}\text { MENOS QUE } \\
\text { ADEQUADO }\end{array}$ & $0-2$ & $0-10$ & $0-6$ & $0-6$ & $0-27$ \\
\hline & $\begin{array}{l}\text { MODERADAMENTE } \\
\text { ADEQUADO }\end{array}$ & $3-4$ & $11-12$ & $7-9$ & $7-8$ & $28-33$ \\
\hline & ADEQUADO & 5 & $13-14$ & $10-14$ & $9-11$ & $34-40$ \\
\hline & EXCELENTE & $6-7$ & $15-20$ & $15-22$ & $12-18$ & $41-67$ \\
\hline
\end{tabular}

\section{Categorias Descritivas:}

Menos que adequado: As oportunidades (affordances) que o ambiente oferece ao desenvolvimento motor do bebê estão faltando (são poucas) ou precisam ser melhoradas. Sugestão: A família deve procurar adicionar ou melhorar certas dimensões do ambiente domiciliar, oferecendo variedade de oportunidades que podem beneficiar o desenvolvimento motor do seu bebê.

Moderadamente adequado: O ambiente oferece algumas oportunidades (affordances) para o desenvolvimento motor, porém, estas poderiam ser melhoradas. Sugestão: Acrescentar mais oportunidades que podem ajudar a estimular o desenvolvimento motor do seu bebê.

Adequado: $\mathrm{O}$ ambiente mostra suficiente quantidade e qualidade de opportunidades (affordances). Sugestão: Continuar usando e buscando formas diferentes de explorar as opportunidades que o lar pode proporcionar ao desenvolvimento motor do seu bebê.

Excelente: O ambiente domiciliar oferece ampla quantidade e variedade de oportunidades (affordances). Sugestão: Continuar usando e procurando formas diferentes de encorajar o movimento e brincadeiras com o seu bebê.

Alguns exemplos de como usar o AHEMD - Escala Bebê para melhor o ambiente do lar para o desenvolvimento motor:

Ao final da avaliação, você terá 5 fatores para interpretar: 4 dimensões (Espaço Físico, Variedade de Estimulação, Brinquedos de Motricidade Fina, Brinquedos de Motricidade Grossa) e a Pontuação Total.

Os resultados das categorias descritivas vão ajudar no entendimento de como cada dimensão se comporta em relação à pontuação total do ambiente. Por exemplo, uma ou duas dimensões com pontuação baixa podem contribuir para uma pontuação total baixa. Se esse for o caso, é importante focar nestas dimensões para melhorar as oportunidades para o desenvolvimento motor do bebê.

${ }^{a}$ C Esse questionário foi desenvolvido pelo Developmental Motor Cognition Lab - University of Texas at Arlington (USA), Motor Development Lab - Texas A\&M University (USA) e Laboratório de Pesquisa em Desenvolvimento Neuromotor - Universidade Metodista de Piracicaba (Brasil). Todos os direitos reservados. 
Além da análise por dimensão, é muito importante verificar os itens específicos dentro de cada dimensão para determinar quais são os aspectos do lar que devem ser adicionados ou modificados para melhorar oportunidades de ação para o bebê.

Exemplos para melhorar cada dimensão são:

- Espaço Físico: Se há uma falta de tipos de piso/solo diferenciados (questão 2) ou de degraus/escadas (questões 5 e 7) no espaço interior e exterior, leve o bebê para lugares fora da casa que possam oferecer tais oportunidades;

- Variedade de Estimulação: A falta de brincadeiras que encorajam aprender sobre as partes do corpo (questão 9) ou a falta de costume de colocar o bebê para brincar deitado de barriga para baixo (questão 14), podem ser facilmente incluídos na rotina diária do bebê;

- Brinquedos de Motricidade Fina: A falta de brinquedos musicais (questão 21) ou de livros para bebês (questão 26) pode direcionar os pais na próxima vez que forem comprar um novo brinquedo;

- Brinquedos de Motricidade Grossa: A falta de balanços para bebês (questão 29) pode ser compensada, levando o bebê a lugares fora de casa que têm esse tipo de brinquedo e expor a criança a tais oportunidades. A falta de um tapete emborrachado (questão 20) pode ser compensada por uma superfície confortável (um colchonete ou acolchoado no chão) que permita ao bebê ser mais ativo (rolar, engatinhar, etc.).

Observações: 